\title{
APPROXIMATION ORDER FROM CERTAIN SPACES OF SMOOTH BIVARIATE SPLINES ON A THREE-DIRECTION MESH ${ }^{1}$
}

\author{
RONG-QING JIA
}

\begin{abstract}
Let $\Delta$ be the mesh in the plane obtained from a uniform square mesh by drawing in the north-east diagonal in each square. Let $\pi_{k, \Delta}^{\rho}$ be the space of bivariate piecewise polynomial functions in $C^{\rho}$, of total degree $\leq k$, on the mesh $\Delta$. Let $m(k, \rho)$ denote the approximation order of $\pi_{k, \Delta}^{\rho}$. In this paper, an upper bound for $m(k, \rho)$ is given. In the space $3 \leq 2 k-3 \rho \leq 7$, the exact values of $m(k, \rho)$ are obtained:

$$
\begin{array}{ll}
m(k, \rho)=2 k-2 \rho-1 & \text { for } 2 k-3 \rho=3 \text { or } 4, \\
m(k, \rho)=2 k-2 \rho-2 & \text { for } 2 k-3 \rho=5,6 \text { or } 7 .
\end{array}
$$
\end{abstract}

In particular, this result answers negatively a conjecture of de Boor and Höllig.

1. Introduction. In this paper we study the approximation order from certain spaces of smooth bivariate splines on a three-dimension mesh. The work in this respect has been initiated by de Boor and DeVore [BD], and de Boor and Höllig $\left[\mathbf{B H}_{\mathbf{1}}-\mathbf{B H}_{3}\right]$. Here we follow them and introduce some notation. Let

$$
\Delta:=\bigcup_{n \in Z}\left\{\left(x_{1}, x_{2}\right) \in R^{2} ; x_{1}=n, x_{2}=n \text { or } x_{2}-x_{1}=n\right\} .
$$

Namely, the mesh $\Delta$ is obtained from a uniform square mesh by drawing in the north-east diagonal in each square. Let

$$
S:=\pi_{k, \Delta}^{\rho}:=\pi_{k, \Delta} \cap C^{\rho}
$$

be the space of bivariate pp (piecewise polynomial) functions in $C^{\rho}$, of total degree $\leq k$, on the mesh $\Delta$. The approximation order of $S$ is, by definition, the integer $m$ for which the following holds: For any $f \in C^{m}$,

$$
\operatorname{dist}\left(f, S_{h}\right) \leq(\text { const }) h^{m}|f|_{m, \infty}
$$

while, for some $C^{m+1}$-function $f$ with $\|f\|_{m+1, \infty}<\infty$,

$$
\operatorname{dist}\left(f, S_{h}\right) \neq o\left(h^{m}\right) \text {. }
$$

Here the scale $\left(S_{h}\right)$ of approximating spaces is generated from $S$ by simple scaling:

with

$$
S_{h}:=\sigma_{h}(S)
$$

$$
\left(\sigma_{h} f\right)(x):=f(x / h), \text { all } f, x, h .
$$

Received by the editors November 2, 1984 and, in revised form, June 6, 1985.

1980 Mathematics Subject Classification. Primary 41A20.

${ }^{1}$ This material is based upon work supported by the National Science Foundation under Grant No. MCS-8210950. This was also sponsored by the United States Army under Contract No. DAAG29-80-C-0041. 
Further,

$$
\operatorname{dist}(f, S):=\inf _{s \in S}\|f-s\|
$$

and $\|\cdot\|$ is the sup norm on $\mathbf{R}^{2}$ :

$$
\|f\|:=\|f\|_{\infty}:=\sup _{x \in R^{2}}|f(x)| .
$$

Moreover,

$$
|f|_{m, \infty}:=\sum_{|\alpha|=m}\left\|D^{\alpha} f\right\|, \quad\|f\|_{m, \infty}:=\sum_{|\alpha| \leq m}\left\|D^{\alpha} f\right\| .
$$

We denote by $m(k, \rho)$ the approximation order of $\pi_{k, \Delta}^{\rho}$.

Only a few results about $m(k, \rho)$ are known:

$$
\begin{gathered}
m(k, \rho)= \begin{cases}k+1 & \text { for } k \geq 4 \rho+1(\text { see }[\mathbf{B Z}]), \\
0 & \text { for } 2 k-3 \rho \leq 1(\text { see }[\mathbf{B D}]) \\
2 k-2 \rho & \text { for } 2 k-3 \rho=2(\text { see }[\mathbf{B H}])\end{cases} \\
m(3,1)=3 \quad\left(\text { see }\left[\mathbf{B H} \mathbf{H}_{\mathbf{2}}\right]\right)
\end{gathered}
$$

An upper bound for $m(k, \rho)$ has been obtained by de Boor and Höllig (see $\left[\mathbf{B H}_{\mathbf{3}}\right.$, Theorem 3]):

$$
m(k, \rho) \leq \min \{2(k-\rho), k+1\} .
$$

Also, they raised the following

CONJECTURE. $m(k, \rho) \geq \min \{2(k-\rho), k+1\}-1$.

By using a quasi-interpolant scheme, $[\mathbf{J}]$ gives

$$
m(k, \rho) \geq \min \{2(k-\rho), k+1\}-2 .
$$

A question naturally arises: Can the lower bound given by (1) be improved? This paper shows that this lower bound is sharp. More precisely, we will prove the following results:

$$
\begin{gathered}
m(k, \rho)=2 k-2 \rho-1 \quad \text { for } 2 k-3 \rho=3 \text { or } 4 \\
m(k, \rho)=2 k-2 \rho-2 \quad \text { for } 2 k-3 \rho=5,6 \text { or } 7
\end{gathered}
$$

In particular,

$$
m(k, \rho)=\min \{2(k-\rho), k+1\}-2 \text { for } 2 k-3 \rho=5,6 \text { or } 7 \text { and } k \leq 2 \rho+1 .
$$

This answers negatively the conjecture of de Boor and Höllig.

Here is an outline of this paper. $\S \S 2-4$ treat the algebra generated by the shift operators, the box splines and the jump operators, respectively. Those three sections are tools, and they prepare for the core of this paper, $\S 5$, which reduces the approximation problem to a determinant problem and hence gives an upper bound for the approximation order. In $\S 6$, the result of $\S 5$ is applied to obtain (2) and (3).

Before proceeding with the proofs of (2) and (3), we need to introduce more notation. For a set $E$, we denote by $|E|$ the cardinality of $E$. Let $\mathbf{Z}_{+}$be the set of nonnegative integers. Let $e_{1}, e_{2}$ be the unit coordinate vectors in the plane; i.e.,

$$
e_{1}=(1,0) \quad e_{2}=(0,1)
$$

As usual, $D_{i}$ denotes the derivative with respect to the $i$ th argument $(i=1,2)$. Let

$$
e_{3}=e_{1}+e_{2} \text { and } D_{3}=D_{1}+D_{2} \text {. }
$$


For a bivariate function $f: \mathbf{R}^{2} \rightarrow \mathbf{R}$ and a real number $a$, the difference operators $\nabla_{r, a}$ are given by

$$
\nabla_{r, a} f:=f-f\left(\cdot-a e_{r}\right) \quad(r=1,2,3) .
$$

If $a=1, \nabla_{r, a}$ is abbreviated to $\nabla_{r}$. Let $\pi$ be the space of all bivariate polynomials, $\pi_{k}$ the space of all bivariate polynomials of total degree $\leq k$. For a polynomial $p$, its degree is denoted by $\operatorname{deg} p$.

2. The algebra generated by the shift operators. A mapping from $Z^{2}$ to $\mathbf{R}$ is called a bivariate sequence. The set of all the bivariate sequences equipped with addition and scalar multiplication forms a linear space, which we denote by $l\left(\mathbf{Z}^{2}\right)$. All the linear operators on $l\left(\mathbf{Z}^{2}\right)$ form a noncommutative algebra. We want to consider one of its subalgebras. Let $T_{r}$ be the shift operators given by

$$
T_{r} g:=g\left(\cdot-e_{r}\right), \quad \text { all } g \in l\left(\mathbf{Z}^{2}\right)(r=1,2,3) .
$$

Clearly

$$
T_{3}=T_{2} T_{1}=T_{1} T_{2} \text {. }
$$

Let $A$ be the subalgebra generated by $T_{1}, T_{1}^{-1}, T_{2}$ and $T_{2}^{-1}$. Then $A$ is commutative. Let $I$ be the identity operator on $l\left(\mathbf{Z}^{2}\right)$. Then the difference operators can be represented as

$$
\nabla_{r}=I-T_{r} \quad(r=1,2,3)
$$

In particular,

$$
\begin{aligned}
\nabla_{3} & =I-T_{3}=I-T_{1} T_{2} \\
& =I-\left(I-\nabla_{1}\right)\left(I-\nabla_{2}\right)=\nabla_{1}+\nabla_{2}-\nabla_{1} \nabla_{2} .
\end{aligned}
$$

If we impose a sup norm on $l\left(\mathbf{Z}^{2}\right)$, then we get the normed linear space $l_{\infty}\left(\mathbf{Z}^{2}\right)$. Thus we can talk about the norm of an operator on $l_{\infty}\left(\mathbf{Z}^{2}\right)$ in the usual sense. Moreover, we can talk about positive operators. A sequence $g \in l\left(\mathbf{Z}^{2}\right)$ is called positive, and denoted by $g \geq 0$, if

$$
g(j) \geq 0 \text { for any } j \in \mathbf{Z}^{2} .
$$

An operator $L$ is called positive if

$$
L g \geq 0 \text { whenever } g \geq 0 \text {. }
$$

A sequence $g \in l\left(\mathbf{Z}^{2}\right)$ is called constant if there exists a real number $b$ such that

$$
g(j)=b \quad \text { for any } j \in \mathbf{Z}^{2} \text {. }
$$

We denote by 1 the constant sequence which takes value 1 . If $L$ is a positive operator on $l\left(\mathbf{Z}^{2}\right)$, then we have $\|L\|=\|L \mathbf{1}\|$. Indeed, since $-\|g\| \mathbf{1} \leq g \leq\|g\| \mathbf{1}$, we have

and so

$$
-\|g\|(L \mathbf{1}) \leq L g \leq\|g\|(L \mathbf{1})
$$

$$
\|L g\| \leq\|g\|\|L \mathbf{1}\| \text {. }
$$

This shows that $\|L\| \leq\|L \mathbf{1}\|$. The other direction holds because $\|\mathbf{1}\|=1$. To give an example of positive operators, we consider

$$
H_{r}:=\sum_{t=0}^{N-1} T_{r}^{t} \quad(r=1,2,3),
$$


where $N$ is a positive integer. Then $H_{r} \mathbf{1}=N$; hence

$$
\left\|H_{r}\right\|=N \text { for } r=1,2,3 .
$$

3. Box splines. Box splines were introduced in [BD and $\left.\mathbf{B H}_{\mathbf{1}}\right]$ and have proved useful in approximation problems. The key point is that the approximation order of $S$ is determined by all the box splines contained in $S$ (see $\left[\mathbf{B H}_{3}\right]$ ). Here we specify the definition of box splines from $\left[\mathbf{B H}_{\mathbf{1}}\right]$ to suit our discussion. For $\lambda=\left(\lambda_{1}, \lambda_{2}, \lambda_{3}\right) \in \mathbf{Z}_{+}^{3}$ with $|\lambda|=\lambda_{1}+\lambda_{2}+\lambda_{3}$ as usual, let $\Xi=\left(\xi_{i}\right)_{1}^{|\lambda|}$ be the sequence given by

$$
\xi_{1}=\cdots=\xi_{\lambda_{1}}=e_{1}, \quad \xi_{\lambda_{1}+1}=\cdots=\xi_{\lambda_{1}+\lambda_{2}}=e_{2}, \quad \xi_{\lambda_{1}+\lambda_{2}+1}=\cdots=\xi_{|\lambda|}=e_{3}
$$

Then the box spline $M_{\lambda}:=M_{\Xi}$ is defined as the distribution given by the rule

$$
M_{\Xi}: \phi \rightarrow \int \phi\left(\sum_{i=1}^{|\lambda|} u(i) \xi_{i}\right) d u
$$

where the integral is taken over the cube $[0,1]^{|\lambda|}$. Let

$$
d:=|\lambda|-\max \left\{\lambda_{r}\right\}-1 \text {. }
$$

Then $M_{\lambda} \subset L_{\infty}^{(d)} \cap C^{(d-1)}$. In addition, $d$ is the largest integer such that this relation is true. In particular, a box spline $M_{\lambda}$ belongs to $L_{\infty}$ if and only if $|\lambda|-\max \left\{\lambda_{r}\right\} \geq$ 1. In what follows, all the box splines are assumed to be in $L_{\infty}$.

A box spline series has the following nice property with respect to derivatives:

$$
D_{i}\left(\sum_{j \in Z^{2}} a(j) M_{\Xi}(\cdot-j)\right)=\sum_{j \in Z^{2}}\left(\nabla_{i} a\right)(j) M_{\Xi \backslash e_{i}}(\cdot-j), \quad \text { if } e_{i} \in \Xi .
$$

Let $S_{\lambda}:=$ the linear span of $M_{\lambda}(\cdot-j), j \in Z^{2}$; that is,

$$
S_{\lambda}:=\left\{\sum a(j) M_{\lambda}(\cdot-j) ; a \in l\left(Z^{2}\right)\right\} .
$$

LEMMA 1. The following inclusion relations hold:

$$
\begin{array}{ll}
S_{\lambda_{1}, \lambda_{2}, \lambda_{3}} \subset S_{\lambda_{1}+1, \lambda_{2}, \lambda_{3}-1}+S_{\lambda_{1}+1, \lambda_{2}-1, \lambda_{3}} & \text { if } \min \left\{\lambda_{2}, \lambda_{3}\right\} \geq 1 ; \\
S_{\lambda_{1}, \lambda_{2}, \lambda_{3}} \subset S_{\lambda_{1}, \lambda_{2}+1, \lambda_{3}-1}+S_{\lambda_{1}-1, \lambda_{2}+1, \lambda_{3}} & \text { if } \min \left\{\lambda_{3}, \lambda_{1}\right\} \geq 1 ; \\
S_{\lambda_{1}, \lambda_{2}, \lambda_{3}} \subset S_{\lambda_{1}-1, \lambda_{2}, \lambda_{3}+1}+S_{\lambda_{1}, \lambda_{2}-1, \lambda_{3}+1} & \text { if } \min \left\{\lambda_{1}, \lambda_{2}\right\} \geq 1 .
\end{array}
$$

PrOOF. We need only prove $\left(1^{\circ}\right)$. Any $s \in S_{\lambda}$ can be expressed as a series $\sum a(j) M_{\lambda}(\cdot-j)$. Set, for $j=\left(j_{1}, j_{2}\right) \in Z^{2}$,

$$
b\left(j_{1}, j_{2}\right):= \begin{cases}a\left(0, j_{2}\right)+\cdots+a\left(j_{1}, j_{2}\right) & \text { for } j_{1} \geq 0 \\ 0 & \text { for } j_{1}=-1 \\ -a\left(-1, j_{2}\right)-\cdots-a\left(j_{1}+1, j_{2}\right) & \text { for } j_{1}<-1\end{cases}
$$

Then $\nabla_{1} b=a$; hence

$$
\begin{aligned}
s & =\sum\left(\nabla_{1} b\right)(j) M_{\lambda}(\cdot-j)=D_{1}\left(\sum b(j) M_{\lambda_{1}+1, \lambda_{2}, \lambda_{3}}(\cdot-j)\right) \\
& =\left(D_{3}-D_{2}\right)\left(\sum b(j) M_{\lambda_{1}+1, \lambda_{2}, \lambda_{3}}(\cdot-j)\right) \\
& =\sum\left(\nabla_{3} b\right)(j) M_{\lambda_{1}+1, \lambda_{2}, \lambda_{3}-1}(\cdot-j)-\sum\left(\nabla_{2} b\right)(j) M_{\lambda_{1}+1, \lambda_{2}-1, \lambda_{3}}(\cdot-j) .
\end{aligned}
$$


This shows that

$$
s \in S_{\lambda_{1}+1, \lambda_{2}, \lambda_{3}-1}+S_{\lambda_{1}+1, \lambda_{2}-1, \lambda_{3}} .
$$

The proof of Lemma 1 is complete.

4. Jump operators. We denote by $\mathbf{S}_{\Delta}$ the space of all splines (piecewise polynomials) on the mesh $\Delta$. For $s \in \mathbf{S}_{\Delta}$, we think of $s$ as defined on $\mathbf{R}^{2} \backslash \Delta$. To describe the jump of a given spline $s$ in the direction $e_{r}(r=1,2,3)$, we introduce the jump operators $J_{r}$ as follows:

$$
J_{r} s:=\lim _{\varepsilon \rightarrow+0}\left[s\left(\cdot+\varepsilon e_{r}\right)-s\left(\cdot-\varepsilon e_{r}\right)\right] .
$$

On each component of $\mathbf{R}^{2} \backslash \Delta, s$ is a polynomial; hence the above limit always exists. Clearly, if $s$ is continuous at $x$, then $J_{r} s(x)=0$ for all $r=1,2,3$. Thus the support of $J_{r} s$ is included in $\Delta$. Since we think of $s$ as defined on $\mathbf{R}^{2} \backslash \Delta, J_{r} s$ is thought of as defined on $\Delta \backslash \mathbf{Z}^{2}$. The operators $J_{r}$ are linear and bounded: $\left\|J_{r}\right\| \leq 2$.

If $g$ is defined on $\Delta \backslash \mathbf{Z}^{2}$, and if $g$ is a polynomial in each component of $\Delta \backslash \mathbf{Z}^{2}$, then we can define

$$
K_{r} g(j):=\lim _{\delta \rightarrow+0} g\left(j+\delta e_{r}\right), \quad j \in Z^{2}, r=1,2,3 .
$$

The operators $K_{r}$ given by the above are linear and bounded: $\left\|K_{r}\right\| \leq 1$.

We also want to give a description for the jump of the derivatives of a given spline. To this end we introduce the operators $R_{r, n}$ on $l\left(\mathbf{Z}^{2}\right)$ given by the rule

$$
R_{r, n} a:=\sum_{t=0}^{n} a\left(\cdot-t e_{r}\right) M_{n}(t), \quad r=1,2,3 ; n \geq 1 .
$$

(Recall that $M_{n}$ is the univariate $B$-spline with support $[0, n]$ on the uniform mesh Z.) When $n<1$, we interpret $R_{r, n}$ as zero. Since $\sum_{t=0}^{n} M_{n}(t)=1$, the operator $R_{r, n}$ is bounded by 1 .

We make some convention about the combination notation $\left(\begin{array}{c}m \\ n\end{array}\right)$. Whatever $m$ and $n$ might be, we agree that

$$
\left(\begin{array}{l}
m \\
n
\end{array}\right)= \begin{cases}1 & \text { if } m=n \\
0 & \text { if } m<n\end{cases}
$$

Now we are ready to state the main result of this section.

LEMMA 2. The following formulae hold:

$$
\begin{aligned}
& K_{1} J_{2} D_{2}^{k-1}\left(\sum a(j) M_{\lambda}(\cdot-j)\right) \\
& =R_{1,|\lambda|-k}\left(\begin{array}{c}
k-\lambda_{2}-1 \\
\lambda_{3}-1
\end{array}\right)\left(-\nabla_{1}\right)^{k-\left(\lambda_{2}+\lambda_{3}\right)} \nabla_{2}^{\lambda_{2}} \nabla_{3}^{\lambda_{3}} a \\
& K_{2} J_{3} D_{3}^{k-1}\left(\sum a(j) M_{\lambda}(\cdot-j)\right) \\
& =R_{2,|\lambda|-k}\left(\begin{array}{c}
k-\lambda_{3}-1 \\
\lambda_{1}-1
\end{array}\right) \nabla_{1}^{\lambda_{1}} \nabla_{2}^{k-\left(\lambda_{3}+\lambda_{1}\right)} \nabla_{3}^{\lambda_{3}} a \\
& K_{3} J_{1} D_{1}^{K-1}\left(\sum a(j) M_{\lambda}(\cdot-j)\right) \\
& =R_{3,|\lambda|-k}\left(\begin{array}{c}
k-\lambda_{1}-1 \\
\lambda_{2}-1
\end{array}\right) \nabla_{1}^{\lambda_{1}}\left(-\nabla_{2}\right)^{\lambda_{2}} \nabla_{3}^{k-\left(\lambda_{1}+\lambda_{2}\right)} a .
\end{aligned}
$$


ProOF. We need only prove $\left(1^{\circ}\right)$, because $\left(2^{\circ}\right)$ and $\left(3^{\circ}\right)$ can be proved in a similar way. For simplicity, write

$$
s=\sum a(j) M_{\lambda}(\cdot-j) .
$$

The proof of $\left(1^{\circ}\right)$ will go case by case.

Case 1. $k \leq \lambda_{2}-1$.

In this case,

$$
D_{2}^{k-1} s=\sum\left(\nabla_{2}^{k-1} a\right)(j) M_{\lambda_{1}, \lambda_{2}-k+1, \lambda_{3}}(\cdot-j) .
$$

Since $\lambda_{2}-k+1 \geq 2, M_{\lambda_{1}, \lambda_{2}-k+1, \lambda_{3}}$ is continuous in the $e_{2}$ direction. This shows that $J_{2} D_{2}^{k-1} s=0$. On the other hand, $k-\lambda_{2}-1<\lambda_{3}-1$; hence the right-hand side of $\left(1^{\circ}\right)$ is also zero in this case.

Case 2. $k=\lambda_{2}$.

This case is divided into three subcases.

Subcase 1. $\min \left\{\lambda_{1}, \lambda_{3}\right\} \geq 1$.

In this case,

$$
D_{2}^{k-1} s=\sum\left(\nabla_{2}^{k-1} a\right)(j) M_{\lambda_{1}, 1, \lambda_{3}}(\cdot-j) .
$$

Since $M_{\lambda_{1}, 1, \lambda_{3}} \in C\left(R^{2}\right)$, we obtain the desired conclusion.

Subcase 2. $\lambda_{1}=0, \lambda_{3} \geq 1$.

In this case, $M_{\lambda}$ is continuous in the $e_{2}$ direction at the points $j+\delta e_{1}, j \in \mathbf{Z}^{2}$, $0<\delta<1$; hence $K_{1} J_{2} D_{2}^{k-1} s=0$, while the right-hand side of $\left(1^{\circ}\right)$ is also zero, because $k-\lambda_{2}-1<\lambda_{3}-1$.

Subcase 3. $\lambda_{1} \geq 1, \lambda_{3}=0$.

In this case

$$
D_{2}^{k-1} s=\sum\left(\nabla_{2}^{k-1} a\right)(j) M_{\lambda_{1}, 1,0}(\cdot-j)
$$

We have

$$
M_{\lambda_{1}, 1,0}\left(x_{1}, x_{2}\right)=M_{\lambda_{1}}\left(x_{1}\right) M_{1}\left(x_{2}\right) .
$$

It follows that, for $i=\left(i_{1}, i_{2}\right) \in Z^{2}$ and $0<\delta<1$,

$$
J_{2} M_{\lambda_{1}, 1,0}\left(i+\delta e_{1}\right)= \begin{cases}M_{\lambda_{1}}\left(i_{1}+\delta\right) & \text { if } i_{2}=0 \\ -M_{\lambda_{1}}\left(i_{1}+\delta\right) & \text { if } i_{2}=1, \\ 0 & \text { otherwise }\end{cases}
$$

Therefore

$$
\begin{aligned}
& \left(K_{1} J_{2} D_{2}^{k-1} s\right)(i) \\
& \quad=\sum_{j_{1} \in Z}\left[\left(\nabla_{2}^{k-1} a\right)\left(j_{1}, i_{2}\right) M_{\lambda_{1}}\left(i_{1}-j_{1}\right)-\left(\nabla_{2}^{k-1} a\right)\left(j_{1}, i_{2}-1\right) M_{\lambda_{1}}\left(i_{1}-j_{1}\right)\right] \\
& \quad=\sum_{j_{1} \in Z}\left(\nabla_{2}^{k} a\right)\left(j_{1}, i_{2}\right) M_{\lambda_{1}}\left(i_{1}-j_{1}\right) \\
& \quad=\sum_{t=0}^{\lambda_{1}}\left(\nabla_{2}^{k} a\right)\left(i_{1}-t, i_{2}\right) M_{\lambda_{1}}(t) \\
& \quad=R_{1,|\lambda|-k} \nabla_{2}^{\lambda_{2}} a(i) .
\end{aligned}
$$

This proves $\left(1^{\circ}\right)$ in this case, since $k-\lambda_{2}-1=-1=\lambda_{3}-1$ and $k-\left(\lambda_{2}+\lambda_{3}\right)=0$. 
Case 3. $k>\lambda_{2}$.

In this case,

$$
D_{2}^{k-1} s=D_{2}^{k-1-\lambda_{2}}\left(\sum\left(\nabla_{2}^{\lambda_{2}} a\right)(j) M_{\lambda_{1}, 0, \lambda_{3}}(\cdot-j)\right) .
$$

By the binomial theorem, we have

$$
D_{2}^{k-1-\lambda_{2}}=\left(D_{3}-D_{1}\right)^{k-1-\lambda_{2}}=\sum_{p=0}^{k-1-\lambda_{2}}\left(\begin{array}{c}
k-1-\lambda_{2} \\
p
\end{array}\right) D_{3}^{p}\left(-D_{1}\right)^{k-1-\lambda_{2}-p}
$$

If $p \geq \lambda_{3}$ or $k-1-\lambda_{2}-p \geq \lambda_{1}$, then

$$
D_{3}^{p}\left(-D_{1}\right)^{k-1-\lambda_{2}-p} M_{\lambda_{1}, 0, \lambda_{3}}(x)=0 \text { for } x \notin \Delta \text {; }
$$

hence

$$
J_{2}\left(D_{3}^{p}\left(-D_{1}\right)^{k-1-\lambda_{2}-p} M_{\lambda_{1}, 0, \lambda_{3}}\right)=0 .
$$

Assume $p<\lambda_{3}$ and $k-1-\lambda_{2}-p<\lambda_{1}$. Then

$$
\begin{aligned}
D_{3}^{p}(- & \left.D_{1}\right)^{k-1-\lambda_{2}-p}\left(\sum_{j}\left(\nabla_{2}^{\lambda_{2}} a\right)(j) M_{\lambda_{1}, 0, \lambda_{3}}(\cdot-j)\right) \\
& =\sum_{j}\left(\left(-\nabla_{1}\right)^{k-1-\lambda_{2}-p} \nabla_{2}^{\lambda_{2}} \nabla_{3}^{p} a\right)(j) M_{\lambda_{1}+\lambda_{2}+p+1-k, 0, \lambda_{3}-p}(\cdot-j) .
\end{aligned}
$$

Note that

$$
M_{\lambda_{1}+\lambda_{2}+p+1-k, 0, \lambda_{3}-p}\left(x_{1}, x_{2}\right)=M_{\lambda_{1}+\lambda_{2}+p+1-k}\left(x_{1}-x_{2}\right) M_{\lambda_{3}-p}\left(x_{2}\right) .
$$

If $\lambda_{3}-p \geq 2$, then $M_{\lambda_{3}-p}$ is continuous everywhere. Moreover, for fixed $\delta, 0<$ $\delta<1$, and $i=\left(i_{1}, i_{2}\right) \in Z^{2}$,

$$
\begin{aligned}
\lim _{\varepsilon \rightarrow+0}[ & M_{\lambda_{1}+\lambda_{2}+p+1-k}\left(i_{1}-i_{2}+\delta e_{1}+\varepsilon e_{2}\right) \\
& \left.-M_{\lambda_{1}+\lambda_{2}+p+1-k}\left(i_{1}-i_{2}+\delta e_{1}-\varepsilon e_{2}\right)\right]=0 .
\end{aligned}
$$

This shows that

$$
J_{2}\left[D_{3}^{p}\left(-D_{1}\right)^{k-1-\lambda_{2}-p}\left(\sum\left(\nabla_{2}^{\lambda_{2}} a\right)(j) M_{\lambda_{1}, 0, \lambda_{3}}(\cdot-j)\right)\right]=0
$$

unless $p=\lambda_{3}-1$ and $k<|\lambda|$. Thus

$$
\begin{aligned}
& J_{2} D_{2}^{k-1} s \\
& \quad=J_{2}\left[\sum\left(\begin{array}{c}
k-\lambda_{2}-1 \\
\lambda_{3}-1
\end{array}\right)\left(-\nabla_{1}\right)^{k-\left(\lambda_{2}+\lambda_{3}\right)} \nabla_{2}^{\lambda_{2}} \nabla_{3}^{\lambda_{3}-1} a(j) M_{|\lambda|-k, 0,1}(\cdot-j)\right] .
\end{aligned}
$$

By straightforward calculation we have

$$
J_{2} M_{|\lambda|-k, 0,1}\left(i+\delta e_{1}\right)= \begin{cases}M_{|\lambda|-k}\left(i_{1}+\delta\right) & \text { if } i_{2}=0 \\ -M_{|\lambda|-k}\left(i_{1}-1+\delta\right) & \text { if } i_{2}=1 \\ 0 & \text { otherwise }\end{cases}
$$

For simplicity, write

$$
b=\left(\begin{array}{c}
k-\lambda_{2}-1 \\
\lambda_{3}-1
\end{array}\right)\left(-\nabla_{1}\right)^{k-\lambda_{2}-\lambda_{3}} \nabla_{2}^{\lambda_{2}} \nabla_{3}^{\lambda_{3}-1} a .
$$


Then the above calculation yields

$$
\begin{aligned}
J_{2} D_{2}^{k-1} s(i) & =\sum_{j_{1} \in Z}\left[b\left(j_{1}, i_{2}\right) M_{|\lambda|-k}\left(i_{1}-j_{1}\right)-b\left(j_{1}, i_{2}-1\right) M_{|\lambda|-k}\left(i_{1}-j_{1}-1\right)\right] \\
& =\sum_{j_{1} \in Z} \nabla_{3} b\left(j_{1}, i_{2}\right) M_{|\lambda|-k}\left(i_{1}-j_{1}\right) \\
& =R_{1,|\lambda|-k} \nabla_{3} b(i) .
\end{aligned}
$$

This proves $\left(1^{\circ}\right)$ in Case 3. The proof of Lemma 2 is complete.

For simplicity, we denote by $U_{k, r, \lambda}$ the operator appearing on the right-hand side of Lemma $2\left(r^{\circ}\right), r=1,2,3$, respectively. Further, let

$$
\begin{aligned}
L_{k, 1, \lambda} & :=\left(\begin{array}{c}
k-\lambda_{2}-1 \\
\lambda_{3}-1
\end{array}\right)\left(-\nabla_{1}\right)^{k-\lambda_{2}-\lambda_{3}} \nabla_{2}^{\lambda_{2}}\left(\nabla_{1}+\nabla_{2}\right)^{\lambda_{3}} \\
L_{k, 2, \lambda} & :=\left(\begin{array}{c}
k-\lambda_{3}-1 \\
\lambda_{1}-1
\end{array}\right) \nabla_{1}^{\lambda_{1}} \nabla_{2}^{k-\lambda_{3}-\lambda_{1}}\left(\nabla_{1}+\nabla_{2}\right)^{\lambda_{3}} \\
L_{k, 3, \lambda} & :=\left(\begin{array}{c}
k-\lambda_{1}-1 \\
\lambda_{2}-1
\end{array}\right) \nabla_{1}^{\lambda_{1}}\left(-\nabla_{2}\right)^{\lambda_{2}}\left(\nabla_{1}+\nabla_{2}\right)^{k-\lambda_{1}-\lambda_{2}} .
\end{aligned}
$$

5. An upper bound for the approximation order. Let $E$ be a finite subset of $\mathbf{Z}_{+}^{3}$. Let $S$ be the span of $\left\{M_{\lambda}(\cdot-j) ; \lambda \in E, j \in \mathbf{Z}^{2}\right\}$. In this section, we will give an upper bound for the approximation order of $S$.

We want to put the operators $U_{k, r, \lambda}$ and $L_{k, r, \lambda}$ into a two-dimensional array. Note that $U_{k, r, \lambda}=0$ if $k \leq d=\min _{\lambda}\left\{\lambda_{1}+\lambda_{2}, \lambda_{2}+\lambda_{3}, \lambda_{3}+\lambda_{1}\right\}-1$ or $k \geq|\lambda|$. Thus the only interesting case is $d<k<|\lambda|$. Assume $|E|=n$. There is a one-to-one mapping from $\{1, \ldots, n\}$ onto $E$. The image of $q$ under this mapping is denoted by $\lambda(q)$. Let

$$
\begin{aligned}
& U_{3(k-d-1)+r, q}:=U_{k, r, \lambda(q)}, \\
& L_{3(k-d-1)+r, q}:=L_{k, r, \lambda(q)}, \quad r=1,2,3 . \\
& R_{3(k-d-1)+r, q}:=R_{r, \lambda(q)-k},
\end{aligned}
$$

We observe that $L_{k, r, \lambda}$ are homogeneous polynomials in $\nabla_{1}$ and $\nabla_{2}$ of degree $k$. Let

$$
\beta_{3(k-d-1)+r}=k, \quad r=1,2,3 .
$$

We are now in a position to prove the main result of this paper.

THEOREM 1. If $3(m-d) \geq n$, and if the determinant of $L=\left(L_{p q}\right)_{p, q=1}^{n}$ is nonzero, then the approximation order of $S$ does not exceed $m$.

Proof. Suppose to the contrary that to any $h>0$ and any $f \in C^{(m+1)}$ with $\|f\|_{m+1, \infty}<\infty$ there corresponds $u_{h} \in S_{h}$ such that $\left\|f-u_{h}\right\| \leq \varepsilon_{h} h^{m}$ with $\varepsilon_{h} \rightarrow 0$ as $h \rightarrow+0$. It follows that

$$
\left\|\sigma_{1 / h} f-\sigma_{1 / h} u_{h}\right\| \leq \varepsilon_{h} h^{m} .
$$

(Recall that $\sigma_{1 / h}$ is a scaling operator. See $\S 1$.) Assume

$$
u_{h}=\sum_{\lambda} \sum_{j} a_{\lambda, h}(j) M_{\lambda}\left(\frac{\dot{\bar{h}}}{h}-j\right) \text {. }
$$


Then

$$
\sigma_{1 / h} u_{h}=\sum_{\lambda} \sum_{j} a_{\lambda, h}(j) M_{\lambda}(\cdot-j) .
$$

Suppose that $f$ is a polynomial on a square $Q$. Then $\sigma_{1 / h} f$ is a polynomial on the square $Q / h$. In each component of $R^{2} \backslash \Delta$ included in this square, $\sigma_{1 / h}\left(f-u_{h}\right)$ is a polynomial, so we can invoke Markov's inequailty and obtain

$$
\left\|D_{2}^{k-1} \sigma_{1 / h} f-D_{2}^{k-1} \sigma_{1 / h} u_{h}\right\| \leq \text { const } \varepsilon_{h} h^{m} .
$$

Moreover, since $K_{1}$ and $J_{2}$ both are bounded operators, we have

$$
\left\|K_{1} J_{2} D_{2}^{k-1} \sigma_{1 / h} f-K_{1} J_{2} D_{2}^{k-1} \sigma_{1 / h} u_{h}\right\| \leq \text { const } \varepsilon_{h} h^{m} .
$$

But $f \in C^{(m+1)}$; hence

$$
K_{1} J_{2} D_{2}^{k-1} \sigma_{1 / h} f=0 \text { for } k \leq m .
$$

Thus

By Lemma 2,

$$
\left\|K_{1} J_{2} D_{2}^{k-1} \sigma_{1 / h} u_{h}\right\| \leq \text { const } \varepsilon_{h} h^{m}
$$

$$
K_{1} J_{2} D_{2}^{k-1} \sigma_{1 / h} u_{h}=\sum_{\lambda} U_{k, 1, \lambda} a_{\lambda, h}
$$

hence

$$
\left\|\sum_{\lambda} U_{k, 1, \lambda} a_{\lambda, h}\right\| \leq \text { const } \varepsilon_{h} h^{m} .
$$

The above estimate is also true for $r=2$ or 3 :

$$
\left\|\sum_{\lambda} U_{k, r, \lambda} a_{\lambda, h}\right\| \leq \text { const } \varepsilon_{h} h^{m} .
$$

Let

$$
a_{q}:=a_{\lambda(q), h}, \quad q=1, \ldots, n
$$

and

$$
\xi_{p}:=\sum_{q=1}^{n} U_{p q} a_{q}
$$

Then (6) reads

$$
\left\|\xi_{p}\right\| \leq \text { const } \varepsilon_{h} h^{m}, \quad p=1, \ldots, n
$$

Let

$$
\mathbf{a}=\left(a_{1}, \ldots, a_{n}\right)^{\tau}, \quad \boldsymbol{\xi}=\left(\xi_{1}, \ldots, \xi_{n}\right)^{\tau} .
$$

Here $\tau$ means "transpose". Equation (7) can be written

$$
U \mathbf{a}=\boldsymbol{\xi}
$$

where $U$ is the matrix $\left(U_{p q}\right)_{p, q=1}^{n}$.

Let $I_{n}$ be the $n \times n$ identity matrix. Let $\operatorname{adj}(U)$ be the adjugate matrix of $U$. Then

$$
U(\operatorname{adj} U)=(\operatorname{adj} U) U=(\operatorname{det} U) I_{n} .
$$


By (9), we have

$$
(\operatorname{det} U) \mathbf{a}=(\operatorname{adj} U) U \mathbf{a}=(\operatorname{adj} U) \boldsymbol{\xi} .
$$

Take $h$ to be $1 / N$, where $N$ is a positive integer. Let $\beta:=\sum_{p=1}^{n} \beta_{p}$. Then $\operatorname{det} U$ has the form

$$
\operatorname{det} U=\sum_{\alpha_{1}+\alpha_{2}=\beta} R_{\alpha_{1}, \alpha_{2}} \nabla_{1}^{\alpha_{1}} \nabla_{2}^{\alpha_{2}}
$$

where $R_{\alpha_{1}, \alpha_{2}} \in A$, the algebra generated by the shift operators (see $\S 2$ ), and $\left\|R_{\alpha_{1}, \alpha_{2}}\right\| \leq$ const. Assume adj $U=\left(V_{p q}\right)_{p, q=1}^{n}$. Then each $V_{p q}$ has the form

$$
V_{p q}=\sum_{\alpha_{1}+\alpha_{2}=\beta-\beta_{p}} R_{\alpha_{1}, \alpha_{2}}^{(p, q)} \nabla_{1}^{\alpha_{1}} \nabla_{2}^{\alpha_{2}}
$$

with $R_{\alpha_{1}, \alpha_{2}}^{(p, q)} \in A$ and $\left\|R_{\alpha_{1}, \alpha_{2}}^{(p, q)}\right\| \leq$ const. Let

$$
W:=h^{\beta} H_{1}^{\beta} H_{2}^{\beta}(\operatorname{det} U),
$$

where $H_{1}$ and $H_{2}$ are the operators defined in (4). We observe that

$$
\nabla_{r} H_{r}=\left(I-T_{r}\right)\left(\sum_{t=0}^{N-1} T_{r}^{t}\right)=I-T_{r}^{N}=\nabla_{r, N} \quad(r=1,2,3) .
$$

Hence

$$
W=\sum_{\alpha_{1}+\alpha_{2}=\beta} R_{\alpha_{1}, \alpha_{2}} h^{\beta} H_{1}^{\beta-\alpha_{1}} H_{2}^{\beta-\alpha_{2}} \nabla_{1, N}^{\alpha_{1}} \nabla_{1, N}^{\alpha_{2}} .
$$

Since $\left\|H_{r}\right\| \leq N$, we have

$$
\left\|h^{\beta} H_{1}^{\beta-\alpha_{1}} H_{2}^{\beta-\alpha_{2}}\right\| \leq(1 / N)^{\beta} N^{2 \beta-\left(\alpha_{1}+\alpha_{2}\right)}=1 .
$$

In addition, $\left\|R_{\alpha_{1}, \alpha_{2}}\right\| \leq$ const and $\left\|\nabla_{1, N}^{\alpha_{1}} \nabla_{2, N}^{\sigma_{2}}\right\| \leq$ const; therefore

$$
\|W\| \leq \text { const. }
$$

Next, we want to estimate Wa. It follows from (10) that

$$
W \mathbf{a}=h^{\beta} H_{1}^{\beta} H_{2}^{\beta}(\operatorname{det} U) \mathbf{a}=h^{\beta} H_{1}^{\beta} H_{2}^{\beta}(\operatorname{adj} U) \boldsymbol{\xi} .
$$

Consider $h^{\beta} H_{1}^{\beta} H_{2}^{\beta} V_{p q}$. We have

$$
h^{\beta} H_{1}^{\beta} H_{2}^{\beta} V_{p q}=\sum_{\alpha_{1}+\alpha_{2}=\beta-\beta_{p}} R_{\alpha_{1}, \alpha_{2}}^{(p, q)} h^{\beta} H_{1}^{\beta-\alpha_{1}} H_{2}^{\beta-\alpha_{2}} \nabla_{1, N}^{\alpha_{1}} \nabla_{2, N}^{\alpha_{2}} .
$$

Note that, for $\alpha_{1}+\alpha_{2}=\beta-\beta_{p}$,

$$
\left\|h^{\beta} H_{1}^{\beta-\alpha_{1}} H_{2}^{\beta-\alpha_{2}}\right\| \leq N^{\beta_{p}} \leq N^{m} .
$$

Also $\left\|R_{\alpha_{1}, \alpha_{2}}^{(p, q)}\right\| \leq$ const and $\left\|\nabla_{1, N}^{\alpha_{1}} \nabla_{2, N}^{\alpha_{2}}\right\| \leq$ const. Therefore

$$
\left\|h^{\beta} H_{1}^{\beta} H_{2}^{\beta} V_{p q}\right\| \leq \text { const } N^{m} \text {. }
$$

This combined with (9) enables us to conclude that

$$
\|W \mathbf{a}\| \leq \text { const } N^{m}\|\boldsymbol{\xi}\| \leq \text { const } \varepsilon_{h} .
$$


We restrict the domain of $\sigma_{1 / h} f$ and $\sigma_{1 / h} u_{h}$ to $\mathbf{Z}^{2}$. Thus they become elements of $l\left(\mathbf{Z}^{2}\right)$. Let $G_{\lambda} \in A$ be defined by the rule

$$
G_{\lambda} a=\sum a(j) M_{\lambda}(\cdot-j)
$$

Since $\sum M_{\lambda}(\cdot-j)=1$, we have

$$
\left\|G_{\lambda}\right\| \leq 1
$$

Recall that $q \rightarrow \lambda(q)$ is a one-to-one map from $\{1, \ldots, n\}$ onto $E$. Let $G_{q}=G_{\lambda(q)}$. Then

$$
\sigma_{1 / h} u_{h}=\sum_{q=1}^{n} G_{q} a_{q}
$$

Substitute (13) into (5). Let $W$ act on both sides of this inequality. Since $\|W\| \leq$ const by (11), we obtain

$$
\left\|W \sigma_{1 / h} f-\sum_{q=1}^{n} G_{q} W a_{q}\right\| \leq \text { const } \varepsilon_{h} h^{m} .
$$

Invoking estimate (12), we have

$$
\left\|\sum_{q=1}^{n} G_{q} W a_{q}\right\| \leq \text { const }\|W a\| \leq \text { const } \varepsilon_{h} .
$$

From the foregoing two inequalities we conclude that

$$
\left\|W \sigma_{1 / h} f\right\| \leq \text { const } \varepsilon_{h} .
$$

Suppose now $\operatorname{det} L \neq 0$. Then in the expression

$$
\operatorname{det} L=\sum_{\gamma_{1}+\gamma_{2}=\beta} C_{\gamma_{1}, \gamma_{2}} \nabla_{1}^{\gamma_{1}} \nabla_{2}^{\gamma_{2}},
$$

there exists some $\left(\delta_{1}, \delta_{2}\right)$ such that $\delta_{1}+\delta_{2}=\beta$ and $C_{\delta_{1}, \delta_{2}} \neq 0$. We can find a function $f \in C^{m+1}$ such that $f$ has compact support and

$$
f\left(x_{1}, x_{2}\right)=x_{1}^{\delta_{1}} x_{2}^{\delta_{2}} /\left(\delta_{1} ! \delta_{2} !\right) \text { for }\left(x_{1}, x_{2}\right) \in[-\alpha, \alpha] \times[-\alpha, \alpha],
$$

where $\alpha$ is a sufficiently large real number.

Recall that

$$
R_{r, n}=\sum_{t=0}^{n} M_{n}(t) T_{r}^{t}
$$

Since $\sum M_{n}(t)=1$, we have

$$
\begin{aligned}
I-R_{r, n} & =\sum_{t=0}^{n} M_{n}(t)\left(I-T_{r}^{t}\right) \\
& =\sum_{t=0}^{n} M_{n}(t) \nabla_{r}\left(I+\cdots+T_{r}^{t-1}\right) .
\end{aligned}
$$

We also have observed that $\nabla_{3}-\left(\nabla_{1}+\nabla_{2}\right)=-\nabla_{1} \nabla_{2}$. Now think of $\operatorname{det} U$ as a polynomial in $\nabla_{1}$ and $\nabla_{2}$. Decompose $\operatorname{det} U$ into homogeneous components. Then 
the above facts tell us that $\operatorname{det} L$ is its component of the lowest degree. Therefore we may write

$$
\operatorname{det} U=\operatorname{det} L+\sum_{\gamma_{1}+\gamma_{2}>\beta} c_{\gamma_{1}, \gamma_{2}} \nabla_{1}^{\gamma_{1}} \nabla_{2}^{\gamma_{2}} .
$$

Let

$$
\gamma=\max \left\{\gamma_{1}+\gamma_{2} ; c_{\gamma_{1}, \gamma_{2}} \neq 0\right\} \text {. }
$$

Since $\sigma_{1 / h} f$ is a monomial of degree $\beta$ on the square $[-N \alpha, N \alpha] \times[-N \alpha, N \alpha]$, and since $\operatorname{det} U-\operatorname{det} L$ is a polynomial in $\nabla_{1}$ and $\nabla_{2}$ of degree bigger than $\beta$, we have

$$
(\operatorname{det} U) \sigma_{1 / h} f=(\operatorname{det} L) \sigma_{1 / h} f \quad \text { on } \mathbf{Z}^{2} \cap[-N(\alpha-\gamma), N(\alpha-\gamma)]^{2} \text {. }
$$

Moreover,

$$
\nabla_{1}^{\gamma_{1}} \nabla_{2}^{\gamma_{2}} \sigma_{1 / h} f=0 \quad \text { if }\left(\gamma_{1}, \gamma_{2}\right) \neq\left(\delta_{1}, \delta_{2}\right)
$$

Hence

$$
(\operatorname{det} L) \sigma_{1 / h} f=c_{\delta_{1}, \delta_{2}} \nabla_{1}^{\delta_{1}} \nabla_{2}^{\delta_{2}} \sigma_{1 / h} f
$$

Furthermore,

$$
\begin{aligned}
H_{1}^{\delta_{1}} H_{2}^{\delta_{2}}(\operatorname{det} L) \sigma_{1 / h} f & =c_{\delta_{1}, \delta_{2}}\left(H_{1} \nabla_{1}\right)^{\delta_{1}}\left(H_{2} \nabla_{2}\right)^{\delta_{2}} \sigma_{1 / h} f \\
& =c_{\delta_{1}, \delta_{2}} \nabla_{1, N}^{\delta_{1}} \nabla_{2, N}^{\delta_{2}} \sigma_{1 / h} f=c_{\delta_{1}, \delta_{2}} \nabla_{1}^{\delta_{1}} \nabla_{2}^{\delta_{2}} f=c_{\delta_{1}, \delta_{2}} .
\end{aligned}
$$

Finally, we obtain

$$
\begin{aligned}
W \sigma_{1 / h} f & =h^{\beta} H_{1}^{\beta} H_{2}^{\beta}(\operatorname{det} U) \sigma_{1 / h} f \\
& =h^{\beta} H_{1}^{\beta-\delta_{1}} H_{2}^{\beta-\delta_{2}}\left(H_{1}^{\delta_{1}} H_{2}^{\delta_{2}}(\operatorname{det} U) \sigma_{1 / h} f\right) \\
& =c_{\delta_{1}, \delta_{2}} h^{\beta} H_{1}^{\beta-\delta_{1}} H_{2}^{\beta-\delta_{2}} \mathbf{1} \\
& =c_{\delta_{1}, \delta_{2}} \quad \text { on }[-N(\alpha-\gamma-2 \beta), N(\alpha-\gamma-2 \beta)]^{2} .
\end{aligned}
$$

Therefore (14) becomes

$$
\left|c_{\delta_{1}, \delta_{2}}\right| \leq \text { const } \varepsilon_{h}
$$

But $c_{\delta_{1}, \delta_{2}}$ does not depend on $h$. Letting $h \rightarrow+0$ in the above inequality, we obtain $c_{\delta_{1}, \delta_{2}}=0$. This contradiction shows that the approximation order of $S$ does not exceed $m$. The proof of Theorem 1 is complete.

6. The approximation order of $\pi_{k, \Delta}^{\rho}$ in the case $3 \leq 2 k-3 \rho \leq 7$. De Boor and Höllig have shown that $\pi_{k, \Delta}^{\rho}$ has the same approximation order that $S_{\text {loc }}$ does. Here

$$
S_{\text {loc }}:=\text { the span of }\left\{M_{\lambda}(\cdot-j) ; M_{\lambda} \in \pi_{k, \Delta}^{\rho} \text { and } j \in Z^{2}\right\}
$$

(see $\left.\left[\mathbf{B H}_{3}\right]\right)$. This fact enables us to apply Theorem 1 to obtain the approximation order of $\pi_{k, \Delta}^{\rho}$ in the case $3 \leq 2 k-3 \rho \leq 7$.

Let

$$
E^{\prime}:=\left\{\lambda ; \rho+2 \leq \min \left\{\lambda_{1}+\lambda_{2}, \lambda_{2}+\lambda_{3}, \lambda_{3}+\lambda_{1}\right\}<|\lambda| \leq k+2\right\} .
$$

Then $M_{\lambda} \in \pi_{k, \Delta}^{\rho}$ is equivalent to $\lambda \in E^{\prime}$. By Lemma 1 , we may reduce $E^{\prime}$ to its subset $E$ such that

$$
S_{\text {loc }}=\text { the span of }\left\{M_{\lambda}(\cdot-j) ; \lambda \in E \text { and } j \in Z^{2}\right\} .
$$

Then we form the matrix $L$ as in $\S 5$ and check whether $\operatorname{det} L \neq 0$. In this way we can prove the following theorem. 
THEOREM 2.

$$
\begin{array}{ll}
m(k, \rho)=2 k-2 \rho-1 & \text { for } 2 k-3 \rho=3 \text { or } 4 . \\
m(k, \rho)=2 k-2 \rho-2 & \text { for } 2 k-3 \rho=5,6 \text { or } 7 .
\end{array}
$$

ProOF. (i) The case $2 k-3 \rho=3$.

In this case $\rho$ must be an odd number. There exists some integer $\mu \geq 1$ such that $\rho=2 \mu-1$. Then $k=3 \mu$ and $2 k-2 \rho-1=2 \mu+1=\rho+2$. By Lemma 1 ,

$$
S_{\mathrm{loc}}=S_{\mu, \mu+1, \mu+1}+S_{\mu+1, \mu, \mu+1} \text {. }
$$

Then $E=\{(\mu, \mu+1, \mu+1),(\mu+1, \mu, \mu+1)\}$ and $n=|E|=2$. It is known from $\left[\mathbf{B H}_{1}\right]$ that $m(k, \rho) \geq \rho+2=2 \mu+1$. We want to prove $m(k, \rho)=2 \mu+1$.

We have, for $m=2 \mu+1$, that

$$
L=\left[\begin{array}{cc}
0 & \nabla_{2}^{\mu}\left(\nabla_{1}+\nabla_{2}\right)^{\mu+1} \\
\nabla_{1}^{\mu}\left(\nabla_{1}+\nabla_{2}\right)^{\mu+1} & 0
\end{array}\right] .
$$

Clearly, $\operatorname{det} L \neq 0$. By Theorem 1 we obtain $m(k, \rho) \leq 2 \mu+1$. Thus

$$
m(k, \rho)=2 \mu+1=2 k-2 \rho-1 \text { in the case } 2 k-3 \rho=3 \text {. }
$$

(ii) The case $2 k-3 \rho=4$.

If $\rho=0$, then $m(2,0)=3$ is a well-known fact. Assume $\rho \geq 1$. There exists an integer $\mu \geq 2$ such that $\rho=2 \mu-2$. Then $k=3 \mu-1$ and $2 k-2 \rho-1=2 \mu+1=$ $\rho+3$. It is known from [DM] that $m(k, \rho) \geq 2 k-2 \rho-1$. We want to prove $m(k, \rho) \leq 2 \mu+1$. By Lemma 1 ,

$$
S_{\mathrm{loc}}=S_{\mu+1, \mu, \mu}+S_{\mu, \mu+1, \mu}+S_{\mu, \mu, \mu+1}+S_{\mu, \mu, \mu} .
$$

For $m=2 \mu+1$, we have

$$
L=\left[\begin{array}{cccc}
\nabla_{2}^{\mu}\left(\nabla_{1}+\nabla_{2}\right)^{\mu} & 0 & 0 & \nabla_{2}^{\mu}\left(\nabla_{1}+\nabla_{2}\right)^{\mu} \\
0 & \nabla_{1}^{\mu}\left(\nabla_{1}+\nabla_{2}\right)^{\mu} & 0 & \nabla_{1}^{\mu}\left(\nabla_{1}+\nabla_{2}\right)^{\mu} \\
0 & 0 & \nabla_{1}^{\mu}\left(-\nabla_{2}\right)^{\mu} & \nabla_{1}^{\mu}\left(-\nabla_{2}\right)^{\mu} \\
\mu\left(-\nabla_{1}\right) \nabla_{2}^{\mu}\left(\nabla_{1}+\nabla_{2}\right)^{\mu} & \nabla_{2}^{\mu+1}\left(\nabla_{1}+\nabla_{2}\right)^{\mu} & \nabla_{2}^{\mu}\left(\nabla_{1}+\nabla_{2}\right)^{\mu+1} & \mu\left(-\nabla_{1}\right) \nabla_{2}^{\mu}\left(\nabla_{1}+\nabla_{2}\right)^{\mu}
\end{array}\right] .
$$

Then

$$
\operatorname{det} L=(-1)^{\mu+1} \nabla_{1}^{2 \mu} \nabla_{2}^{3 \mu}\left(\nabla_{1}+\nabla_{2}\right)^{3 \mu}\left(\nabla_{1}+2 \nabla_{2}\right) \neq 0 .
$$

This shows that

$$
m(k, \rho)=2 k-2 \rho-1 \quad \text { in the case } 2 k-3 \rho=4 .
$$

(iii) The case $2 k-3 \rho=5$.

There exists an integer $\mu \geq 1$ such that $\rho=2 \mu-1$. Then $k=3 \mu+1$ and $2 k-2 \rho-2=2 \mu+2$. It is shown by $[J]$ that $m(k, \rho) \geq 2 k-2 \rho-2$. We want to prove $m(k, \rho) \leq 2 k-2 \rho-2$. By Lemma 1 ,

$$
S_{\text {loc }}=\text { the span of }\left\{M_{\lambda}(\cdot-j) ; \lambda \in E, j \in Z^{2}\right\} \text {, }
$$

where

$$
\begin{aligned}
& E=\{(\mu+2, \mu+1, \mu),(\mu+2, \mu, \mu+1)(\mu+1, \mu+2, \mu), \\
&(\mu+1, \mu, \mu+2),(\mu+1, \mu+1, \mu),(\mu+1, \mu, \mu+1)\} .
\end{aligned}
$$


Let $m=2 \mu+2$. To check whether $\operatorname{det} L$ is nonzero, we may use the following technique to simplify the computation. We observe that each entry of the matrix $L$ is a polynomial of $\nabla_{1}$ and $\nabla_{2}$, so we may assign values to $\nabla_{1}$ and $\nabla_{2}$. Write $L=L\left(\nabla_{1}, \nabla_{2}\right)$. If $\operatorname{det} L(1,1) \neq 0$, then $\operatorname{det} L \neq 0$. Let us now look at $L(1,1)$ :

$$
L(1,1)=\left[\begin{array}{cccccc}
2^{\mu} & 2^{\mu+1} & 0 & 0 & 2^{\mu} & 2^{\mu+1} \\
0 & 0 & 2^{\mu} & 0 & 2^{\mu} & 0 \\
0 & 0 & 0 & (-1)^{\mu} & 0 & (-1)^{\mu} \\
-\mu 2^{\mu} & -(1+\mu) 2^{\mu+1} & 2^{\mu} & 2^{\mu+2} & -\mu 2^{\mu} & -(1+\mu) 2^{\mu+1} \\
2^{\mu} & 0 & (\mu+1) 2^{\mu} & 0 & (\mu+1) 2^{\mu} & 2^{\mu+1} \\
0 & (-1)^{\mu} & 0 & 2 \mu(-1)^{\mu} & (-1)^{\mu+1} & 2 \mu(-1)^{\mu}
\end{array}\right]
$$

By straightforward computation, we conclude that $\operatorname{det} L(1,1) \neq 0$. This shows that

$$
m(k, \rho)=2 k-2 \rho-2 \text { in the case } 2 k-3 \rho=5 .
$$

(iv) The case $2 k-3 \rho=6$ or 7 .

The process goes as before. Since the computation is tedious, we omit the details.

The proof of Theorem 2 is complete.

\section{REFERENCES}

[BD] C. de Boor and R. DeVore, Approximation by smooth multivariate splines, Trans. Amer. Math. Soc. 276 (1983), 775-788.

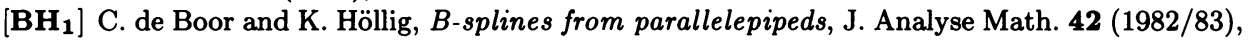
99-115.

[BH $\left.\mathbf{B H}_{2}\right]$, Approximation from piecewise $C^{1}$-cubics: A counterexample, Proc. Amer. Math. Soc. 87 (1983), 649-655.

$\left[\mathbf{B H}_{3}\right]$, Bivariate box splines and smooth pp functions on a three-direction mesh, $\mathrm{J}$. Comput. Appl. Math. 9 (1983), 13-28.

[BZ] J. H. Bramble and M. Zlámel, Triangular elements in the finite element method, Math. Comp. 24 (1970), 809-820.

[DM] W. Dahmen and C. A. Micchelli, On the approximation order from certain multivariate spline spaces, J. Austral. Math. Soc. Ser. B 26 (1984), 233-246.

[J] R. Q. Jia, Approximation by smooth bivariate splines on a three direction mesh, Approximation Theory. IV (Chui, Schumaker and Ward, eds.), Adademic Press, New York, 1983.

Mathematics Research Center, University of Wisconsin, Madison, WisconSIN 53705

Current address: Department of Mathematics, Zhejiang University, Hangzhou, Zhejiang, The People's Republic of China 